\title{
Introduction to Graph-Link Theory
}

\author{
D. P. Ilyutko*, V. O. Manturov
}

\begin{abstract}
The present paper is an introduction to a combinatorial theory arising as a natural generalisation of classical and virtual knot theory. There is a way to encode links by a class of 'realisable' graphs. When passing to generic graphs with the same equivalence relations we get 'graph-links'. On one hand graph-links generalise the notion of virtual link, on the other hand they do not feel link mutations. We define the Jones polynomial for graph-links and prove its invariance. We also prove some a generalisation of the Kauffman-Murasugi-Thistlethwaite theorem on 'minmal diagrams' for graph-links.
\end{abstract}

\section{Introduction}

The discovery of virtual knot theory by Kauffman [Ka1 in mid-1990-s was an important step in generalising combinatorial and topological knot theoretical techniques into a larger domain (knots in thickened surfaces), which is an important step towards generalisation of these techniques for knots in arbitrary manifolds.

It turned out that some invariants (Kauffman bracket polynomial) can be generalised for virtual knots immediately [Ka1], and some other theories (Khovanov homology theory) need a complete revision of the original construction for a generalisation for the case of virtual knots Ma1.

On the other hand, virtual knots sharpened several problems and elicited some phenomena which do not appear in the classical knot theory [FKM], e.g., the existence of a virtual knot with non-trivial Jones polynomial and trivial fundamental group emphasises the difficulty of extracting the Jones polynomial information out of the knot group.

In the present paper, we introduce a new class of objects closely connected to both classical and virtual knots: graph-links. Likewise virtual knots appear out of non-realisable Gauss code and thus generalise classical knots (which have realisable Gauss codes), graphslinks come out of intersection graphs: we may consider graphs which realise chord diagrams, and, in turn, virtual links, and pass to arbitrary simple graphs which correspond

\footnotetext{
*Supported by grants of RF President NSh - 660.2008.1, RFFI 07-01-00648, RNP 2.1.1.7988.
} 
to some mysterious objects generalising links and virtual links. Here we refer to the paper by Traldi and Zulli [TZ] who made a similar work but with another approach: there is a way of coding all knots by Gauss diagrams and there is another way of coding all links by rotating circuits (see, e.g., Ma2]). Thus, the class of objects described in the present paper is larger than the one described by Traldi and Zulli. Rotating circuit approach to both classical and virtual links has several advantages in comparison to the Gauss diagram approach: for classical links, it does not carry nugatory information, it is easier to recognise planarity, and a single circle diagram can encode a link of arbitrarily many components.

On the other hand, while passing from a chord diagram to its intersection graph we forget a lot of information, say, mutant knots are invisible at the level of intersection graphs. Thus, our graph knots are a sort of 'simplified generalised virtual knots'.

Though one can hardly imagine a picture of such a link for a non-realisable graph, in the present paper we constructed an invariant Kauffman bracket corresponding to them and Jones polynomial. This bracket counts the "number of non-existing circles of a nonrealisable chord diagram". The invariance of the bracket agrees with the fact that it does not feel knot mutation.

To construct the Kauffman bracket of a graph-link, one has to count non-existing circles, but to manage with the Khovanov homology, one should also know how these non-existing circles in different Kauffman states of the diagram interfere. In general, the problem seems to be unmanageable (e.g., since the Khovanov homology does detect link mutation, Wehrli [Weh]), but the question is: to which extent one can generalise (or categorify) the Kauffman bracket of a knot. We shall address this question in a sequel of the present paper.

We also use various methods involving Kauffman bracket for detecting the minimal crossing number (minimal vertex number) of a graph-knot, and give many examples.

\section{Preliminaries. Basic constructions}

\subsection{Atoms and virtual links}

First of all, note that in this part of the paper we restrict ourselves to a large class of graph-links corresponding to so-called 'orientable atoms'. Note that classical knots satisfy that condition, i.e. they have an orientable atom. We shall deal with the most general object in a sequel of the present paper.

Definition 2.1. An atom Fom is a pair $(M, \Gamma)$ consisting of a 2-manifold $M$ and a graph $\Gamma$ embedded in $M$ together with a colouring of $M \backslash \Gamma$ in a checkerboard manner. An atom is called orientable if the surface $M$ is orientable. Here $\Gamma$ is called the frame of the atom, whence by genus (atoms and their genera were also studied by Turaev [Tu], and 
atom genus is also called the Turaev genus [Tu] (Euler characteristic, orientation) of the atom we mean that of the surface $M$.

Remark 2.1. Throughout the paper, we shall deal with two types of graphs: four-valent graphs (atom frames or shadows of links) and intersection-type graphs of arbitrary valency. Four-valent graphs are always assumed connected, and the intersection-type graphs may not be connected, though they are assumed to have no loops and no multiple edges.

Having an atom, we may try to embed its frame in $\mathbb{R}^{2}$ in such a way that the structure of opposite half-edges at vertices is preserved. Then we can take the "black angle" structure of the atom to restore the crossings on the plane (as ahead).

In Ma3 it is proved that the link isotopy type does not depend on the particular choice of embedding of the frame into $\mathbb{R}^{2}$ with the structure of opposite edges preserved. The reason is that such embeddings are quite rigid.

The atoms whose frame is embeddable in $\mathbb{R}^{2}$ with opposite half-edge structure preserved are called height or vertical.

However, not all atoms can be obtained from some classical knots. Some abstract atoms may be quite complicated for its frame to be embeddable into $\mathbb{R}^{2}$ with the opposite half-edges structure preserved. However, if it is impossible to embed a graph in $\mathbb{R}^{2}$, we may immerse it by marking artifacts of the immersion (we assume the immersion to be generic) by small circles.

This leads to a connection between atoms and virtual knots which perfectly agrees with virtual knot theory proposed by Kauffman in [Ka1].

Definition 2.2. A virtual diagram is a 4-valent diagram in $\mathbb{R}^{2}$ where each crossing is either endowed with a classical crossing structure (with a choice for underpass and overpass specified) or just said to be virtual and marked by a circle.

Definition 2.3. A virtual link is an equivalence class of virtual link diagrams modulo generalised Reidemeister moves. The latter consist of usual Reidemeister moves referring to classical crossings and the detour move that replaces one arc containing only virtual intersections and self-intersection by another arc of such sort in any other place of the plane, see Fig. 1.

Remark 2.2. Throughout the paper, each virtual diagram has at least one classical crossing.

Remark 2.3. As the detour move does not affect the Kauffman bracket polynomial, we make no difference between virtual diagrams obtained from each other by detours. The coding by chord diagrams and graphs will not see detours at all, so we will be able to check only classical Reidemeister moves to establish any invariance. 

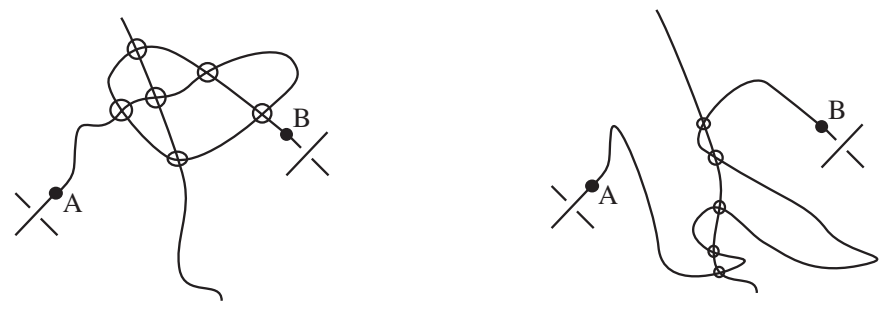

Figure 1: The detour move

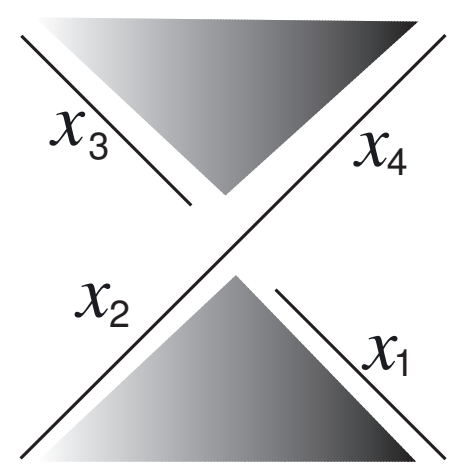

Figure 2: Gluing the cells

Given a virtual diagram $L$, let us construct the atom $A(L)$ out of it. Vertices of $A(L)$ are in one-to-one correspondence with classical crossings of the diagram $L$. Classical crossings of $L$ are connected to each other by branches of $L$ which may intersect each other in virtual crossings. In each classical crossing we have four emanating edges $x_{1}, x_{2}, x_{3}, x_{4}$ in clockwise-ordering such that the pair $\left(x_{1}, x_{3}\right)$ forms an undercrossing and the pair $\left(x_{2}, x_{4}\right)$ forms an overcrossing. These edges are in one-to-one correspondence with edges of $A(L)$ connecting the corresponding vertices. The 1-cycles of the frame pasting black and white cells are as follows. Each boundary of a 2-cell is a rotating circuit on a frame: a circuit which passes every edge at most once and switches at each vertex from an edge to an adjacent (non-opposite) one. Black cells are glued to the angles formed by $\left(x_{1}, x_{2}\right)$ and $\left(x_{3}, x_{4}\right)$, and white cells are glued to the angles formed by $\left(x_{2}, x_{3}\right)$ and $\left(x_{1}, x_{4}\right)$, see Fig. 2. As a result we get an atom.

Let us consider the inverse operation. Having an atom, we may try to construct a virtual knot diagram. To do that, we should take a generic immersion of the atom's frame into $\mathbb{R}^{2}$, put virtual crossings at the intersection points of images of different edges and restore classical crossings at images of vertices 'as above'. Obviously, since we disregard virtual crossings, the most we can expect is the well-definiteness up to detours. However, this allows us to get different virtual link types from the same atom, since for every vertex 


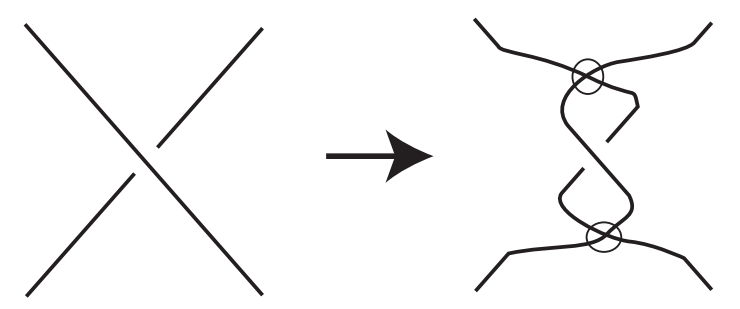

Figure 3: Virtualisation

of the atom with four emanating half-edges $a, b, c, d$ (ordered cyclically on the atom) we may get two different clockwise-orderings on the plane of embedding, $(a, b, c, d)$ and $(a, d, c, b)$. This leads to a move called virtualisation.

Definition 2.4. By a virtualisation of a classical crossing of a virtual diagram we mean a local transformation shown in Fig. 3 .

The above statements summarise as

Proposition 2.1. (see, e.g., $\mathrm{Ma} 4$ ). Let $L_{1}$ and $L_{2}$ be two virtual links obtained from the same atom by using different immersions of its frame. Then $L_{1}$ differs from $L_{2}$ by a sequence of (detours and) virtualisations.

Note that many famous invariants of classical and virtual knots (Kauffman bracket, Khovanov homology [Kh, Ma1, Khovanov-Rozansky homology [KhR1, KhR2]) do not change under the virtualisation, which supports the virtualisation conjecture: if for two classical links $L$ and $L^{\prime}$ there is a sequence $L=L_{0} \rightarrow \cdots \rightarrow L_{n}=L^{\prime}$ of virtualisations and generalised Reidemeister moves then $L$ and $L^{\prime}$ are classically equivalent (isotopic).

Note that the usual virtual equivalence implies classical equivalence for classical links, see GPV]. This means that classical links constitute a proper subset of virtual links.

\subsection{Thickened surface interpretation of virtual links}

Virtual links, being defined diagrammatically, have a topological interpretation. They correspond to links in thickened surfaces $S_{g} \times I$ with fixed $I$-bundle structure up to stabilisations/destabilisations. Projecting $S_{g}$ to $\mathbb{R}^{2}$ (with the condition, however, that all neighbourhoods of crossings are projected with respect to the orientation, we get a diagram on $\mathbb{R}^{2}$ from a generic diagram on $S_{g}$ ): besides the usual crossings arising naturally as projections of classical crossings, we get virtual crossings, which arise as artefacts of the projection: two strands lie in different places on $S_{g}$ but they intersect on the plane because they are forced to do so. 

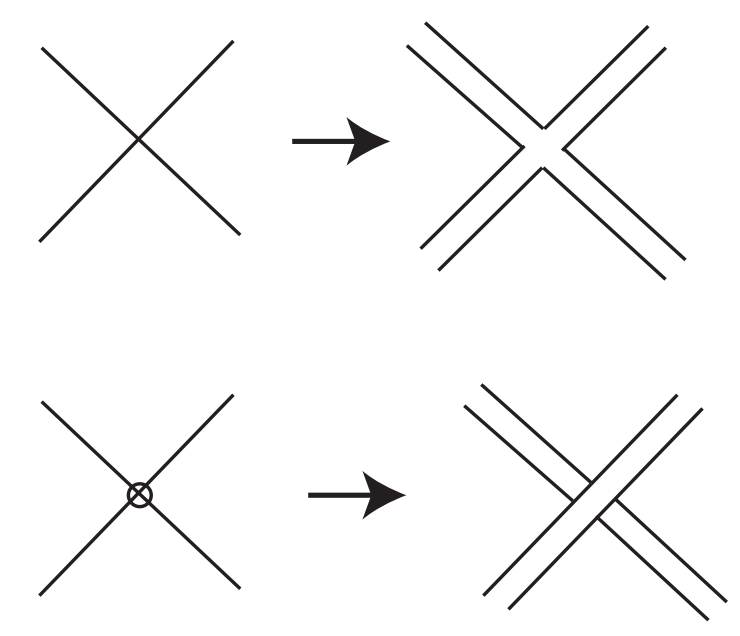

Figure 4: The local structure

We shall use the following construction. Having a virtual link diagram $K$, we take all classical crossings of it and associate with a neighbourhood of a crossing two crossing bands - a 'piece of 2-surface', as shown in Fig. 4, and with a virtual crossing we associate a pair of skew bands, as shown in the lower picture of Fig. 4 .

If we connect these crossings and bands by (non-overtwisted) bands going along edges, we get a 2-surface with boundary. Gluing its boundary components by discs, we get an orientable surface $M(K)$. There is an obvious projection of (a part of) $M(K)$ to $\mathbb{R}^{2}$ in such a way that classical crossings of the link diagram drawn in $M(K)$ generate the diagram of $K$ (with virtual crossings at intersections of skew bands). Then we get a link in $M(K) \times I$ representing the virtual link $K$

Remark 2.4. Note that a thickened surface interpretation differs from the presentation by atoms (see ahead): an atom need not be orientable, while a checkerboard surface need not be 4-colourable. On the other hand, atom does not feel virtualisation, whence the thickened surface does. Later, we will consider only connected thickened surfaces and call the genus of it the underlying genus of a representative for a virtual knot $K$.

For more details, see, e.g., [Ma4].

\subsection{Chord diagrams and intersection graphs}

Definition 2.5. A chord diagram is a graph consisting of a selected oriented cycle (the circle) and several non-oriented edges (chords) connecting points on the circle in such a way that every point is incident to at most one chord. A chord diagram is labeled if 
every chord is endowed with a sign ' + ' or '-'. If no signs are indicated, we assume the chord diagram has all chords with sign ' + '. A labeled chord diagram is a d-diagram if the corresponding intersection graph is bipartite. Two chords in a chord diagram are called linked if the ends of one chord lie in different connected components of the circle without the end-points of the second chord.

Some remarks are in order.

Remark 2.5. Note that $d$-diagrams are precisely those encoding classical link diagrams Ma3]: a chord diagram is embeddable in $\mathbb{R}^{2}$ iff it is a $d$-diagram, and embeddability of a chord diagram yields the planarity of the corresponding four-valent graph (a shadow of the link, which thus has no virtual crossings).

Remark 2.6. To avoid confusion between framing and labeling throughout the paper, we consider only labeled chord diagram with positive framing as each virtual link corresponds to an orientable atom and we forget about framing.

Remark 2.7. In fact, chord diagrams with all positive chords encode all orientable atoms with one white cell: this white cell corresponds to the only $A$-state of the virtual diagram, and chords show how this cell approaches itself in neighbourhoods of crossings (atom vertices). If we want to deal with all atoms and restrict ourselves for the case of one circle, we should take this circle to be corresponding to some other state of the atom, which is encoded by labelings of the chords.

Having a labeled chord diagram $D$, one can construct a virtual link diagram $K(D)$ (up to virtualisation) as follows. Let us immerse this diagram in $\mathbb{R}^{2}$ taking an embedding of the circle and placing some chords inside the circle and the other ones outside the circle. After that we remove a neighbourhoods of each of the chord ends and replace it by a couple of lines with a classical crossing in the following way. A crossing can be smoothed in two ways: $A$ and $B$ as in the Kauffman bracket polynomial. We require that the initial piece of the circle corresponds to the $A$-smoothing if the chord is positive and to the $B$-smoothing if it is negative: $A:\rangle \rightarrow\rangle, B:\rangle \rightarrow \mathcal{Y}$. Note that from $d$-diagrams we get classical links.

Conversely, having a connected virtual diagram $K$ (having at least one classical crossing) with oriented atom, one gets a labeled chord diagram. Indeed, one takes a circuit of $K$ which is a map from $S^{1}$ to the shadow of $K$ which is bijective outside classical and virtual crossings having exactly two inverse images at each classical crossing and two virtual crossings, going transversally at each virtual crossings, and turning from an edge to an adjacent (non-opposite) edge at each classical crossing. This defines a chord diagram (orientation of the circle is chosen arbitrarily), where the sign of the chord is ' + ' if the circuit locally agrees with the $A$-smoothing, and '-' if it agrees with the $B$-smoothing. 
It can be easily checked that this operation is indeed inverse to the operation of constructing a virtual link out of a chord diagram: if we take a chord diagram $D$, and construct a virtual diagram $K(D)$ out of it, then for some circuit the chord diagram corresponding to $K(D)$ will coincide with $D$. The rule for setting classical crossings here agrees with the rule described above because of the orientability of the atom.

This proves the following

Theorem 2.1 ([Ma4]). Any connected virtual diagram with an orientable atom is obtained from a certain labeled chord diagram.

We restrict our attention to connected virtual diagrams with an orientable atoms. The following two theorems show that the set of all such diagrams gives us another class which is wider than the class of classical links but narrower than the one of all virtual links.

As the goal of our paper is to construct the Kauffman bracket polynomial and the Jones polynomial, we disregard virtualisation.

Theorem 2.2. Each two equivalent (in the class of all virtual diagrams) connected virtual diagrams are equivalent in the class of connected virtual diagrams.

Sketch of the proof. Having a disconnected diagram, we can make it connected by adding crossings by second Reidemeister moves. After performing a necessary move, we may perform the inverse Reidemeister move (and, possibly, perform another move in another place). It is easy to see that the process can be organised in such a way that the "auxiliary" crossings do not affect the original moves we have to perform, and in the final diagram all auxiliary crossings are removed. This completes the proof.

Theorem 2.3. Each two equivalent (in the class of all virtual diagrams) virtual diagrams $K_{1}$ and $K_{2}$ with orientable atoms are equivalent in the class of virtual diagrams with orientable atoms.

This theorem was independently proved by O. Ya. Viro and the second named author of the present paper, but the proof was not published. This theorem signifies the consistency of the class of objects we are dealing with: virtual links with orientable atoms constitute a proper part of all virtual links.

Proof. Consider a thickened surface presentation of virtual links, [Ka1, Kup. It can be easily checked that the orientability of the atom corresponding to a virtual link diagram depends only on the $\mathbb{Z}_{2}$-homology class of the corresponding link surface presentation. Consequently, if we perform a move which does not change the genus of the corresponding surface, we do not change orientability of the atom. Obviously, the detours do not affect the surface presentation and hence orientability of the atom. A straightforward check shows neither the first nor the third Reidemeister move changes the genus of the underlying 
surface, thus, does affect the orientability of the atom. The second Reidemeister move may either increase the genus or decrease the genus or leave it as it is. For the case of the second Reidemeister move not changing the genus, the orientability is not changed. Moreover, we may pass from an orientable atom to an orientable atom only in the case when we increase the genus of the thickened surface representation by a second Reidemeister move. Now, assume $K_{1}$ and $K_{2}$ are virtual diagrams with orientable atoms. We use the same notation for the corresponding knots in thickened surfaces: $K_{1}$ and $K_{2}$. It follows from Kuperberg's theorem that there is the following sequence of transformations: we first transform $K_{1}$ to its minimal representative $K_{1}^{\prime}$ having minimal genus. Then we transform $K_{2}$ to its minimal representative $K_{2}^{\prime}$ which has the same genus as $K_{1}^{\prime}$ and is isotopic inside the surface of that genus to $K_{1}^{\prime}$. The latter means that $K_{1}^{\prime}$ and $K_{2}^{\prime}$ are connected by a sequence of genus-preserving Reidemeister moves. So, since both $K_{1}$ and $K_{2}$ have orientable atoms, the whole chain of diagrams $K_{1} \rightarrow \cdots \rightarrow K_{1}^{\prime} \rightarrow \cdots \rightarrow K_{2}^{\prime} \rightarrow \cdots \rightarrow K_{2}$ corresponds to a sequence of orientable atoms, which completes the proof.

The Reidemeister moves give a combinatorial description of the relationship between the different diagrams of a given virtual link. The following definition describes the set of moves corresponding to usual set of Reidemeister moves for planar diagrams. After that we next pass on to moves on graphs.

Definition 2.6. $\Omega 1$. The first Reidemeister chord-move is an addition/removal of an isolated chord labeled '+' or '-' (an isolated chord, not linked with any others).

$\Omega 2$. The second Reidemeister chord-move is an addition/removal of two parallel chords labeled ' + ' and ' - ', so that these chords have the same linked with others.

$\Omega 3$. The third Reidemeister chord-move is shown in Fig. 5. All the chords except three chords shown in Fig. 5 are fixed and their ends lie on the 'dotted' parts of the circle.

$\Omega 4$. The fourth chord-move is shown in Fig. 6. The move takes four segments of the chord diagram denoted by $A, B, C, D$ and transforms the diagram as follows. We perform the surgery along the chords with signs $a$ and $b, a, b \in\{ \pm 1\}$. This surgery cuts the diagram into four pieces $A, B, C, D$ each containing some chord ends, and reconnects them by a pair of vertical lines and a pair of horizontal lines (middle picture). The two chords we are operating with change their labels. After that we redraw the figure to get a 'round circle' of the chord diagram (rightmost picture) and get a shuffle of the segments $A, B, C, D$ with all chord ends lying on them.

Remark 2.8. Using the second Reidemeister and fourth chord-moves, we can replace each chord labeled ' - ' by three chords labeled ' + ' as shown in Fig. 7, Let us call this transformation the fifth move and denote it by $\Omega 5$. The chords having their ends on the 'dotted' parts of the circle are fixed. 

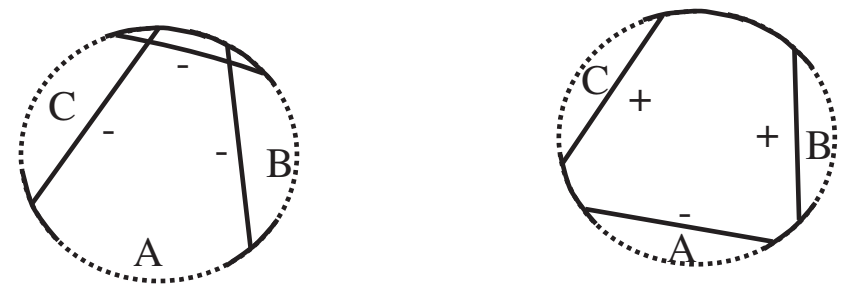

Figure 5: The third Reidemeister chord-move
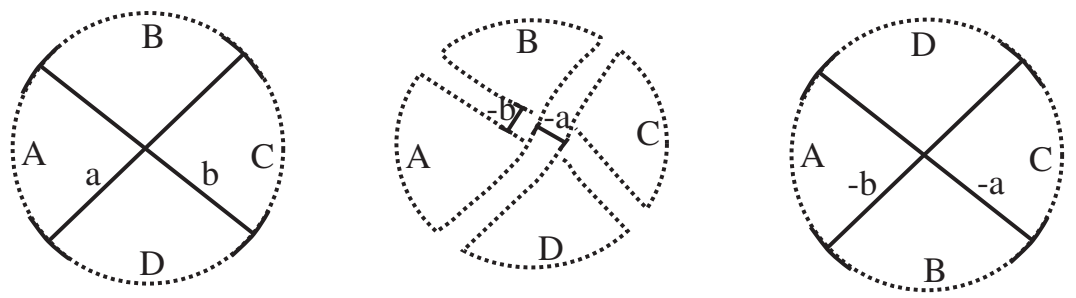

Figure 6: The fourth chord-move
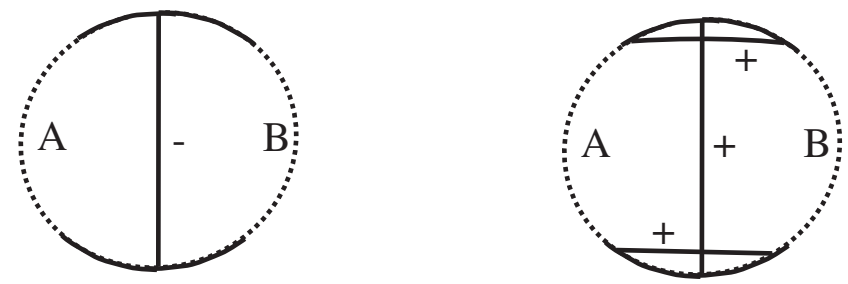

Figure 7: The fifth chord-move 
Theorem 2.4. Let $K_{1}$ and $K_{2}$ be two connected virtual diagrams with orientable atoms, and $D_{1}$ and $D_{2}$ be two labeled chord diagrams obtained from $K_{1}$ and $K_{2}$, respectively. If $K_{1}$ and $K_{2}$ are equivalent in the class of connected diagrams with orientable atoms then $D_{1}$ is obtained from $D_{2}$ by $\Omega 1-\Omega 4$ chord-moves.

Proof. First of all, note that the chord diagrams of two virtual links $K_{1}$ and $K_{2}$ distinguished from each other by the detour moves are the same.

Secondly, when we construct a chord diagram from a virtual diagram $K$ we fix a circuit of $K$ which is a map from $S^{1}$ to the shadow of $K$. The independence from the choice of a circuit is guaranteed by the fourth chord-move $\Omega 4$. Indeed, assume two labeled chord diagrams $D_{1}$ and $D_{2}$ represent the same virtual diagram (up to detours). Then $D_{1}$ and $D_{2}$ can be obtained from each other by a change of rotating circuit. We have to rotate the circuit for $D_{1}$ at some collection of $m$ vertices (chords of $D_{1}$ ) to get the circuit for $D_{2}$. It is easy to see that there is a couple of linked chords among them (otherwise there is no circuit, but a collection of circuits after a surgery). Take a pair of intersecting chords and change the circuit respectively. This will be precisely one fourth move. We get a diagram $D_{1}^{\prime}$ which differs from $D_{2}$ at some collection of $m-2$ vertices. Reiterating the process above, we get to $D_{2}$ from $D_{1}$ in a finite number of steps.

Without loss of generality, we can assume that $K_{1}$ and $K_{2}$ are distinguished from each other by classical Reidemeister moves, for each $K_{1}$ and $K_{2}$ we have rotating circuits of $K_{1}$ and $K_{2}$, and all the moves are performed in the class of connected virtual diagrams with orientable atoms.

Under such assumptions the classical first and second Reidemeister moves on virtual link are equivalent to the first and second Reidemeister chord-moves.

As for the third Reidemeister move on virtual diagrams, it is sufficient to consider only one variant provided that we have the whole collection of the first and second Reidemeister moves, see Oht. At the level of chord diagrams, it is again sufficient to consider only one way of representing this third Reidemeister move (shown in Fig. 8) provided that we have all second Reidemeister chord-moves and the fourth chord-move. Under such assumptions the one classical third Reidemeister move on virtual link is equivalent to the third Reidemeister chord-move, Fig. 8. This completes the proof.

Assume we are given a labeled chord diagram $D$. Let us construct the labeled intersection graph, see [CDL], $G(D)$ as follows. This is the labeled graph whose vertices are in one-to-one correspondence with chords of the diagram, the label of each vertex corresponding to a chord coincides with that of the chord, and two vertices are connected by an edge if and only if the corresponding chords are linked.

Definition 2.7. Let $G$ be a labeled graph on $n$ vertices. Fix an enumeration of vertices for $G$. We define the adjacency matrix $A(G)$ as follows. Set $A(G)=\left(a_{i j}\right)_{i, j=1, \ldots, n}$ be the 

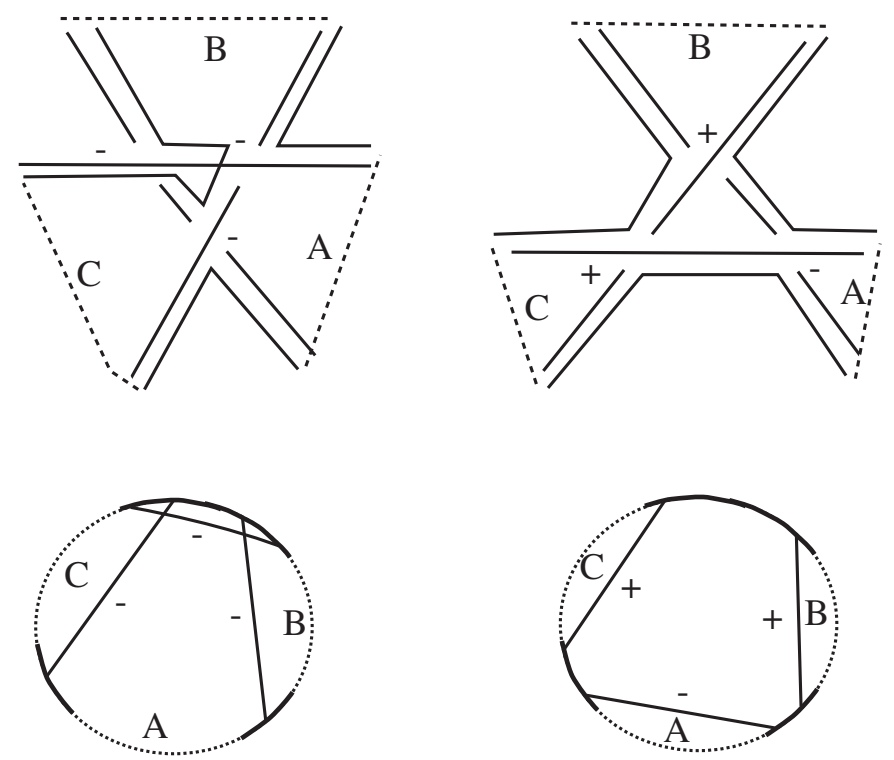

Figure 8: The third Reidemeister move and the third chord-move

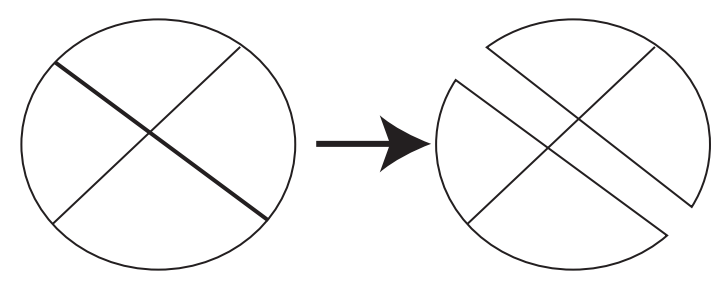

Figure 9: A surgery of the circuit along a chord

adjacency matrix of $G$, i.e. $a_{i j}=1$ if and only if the vertices $i$ and $j$ are incident, and $a_{i j}=0$ otherwise. Besides, we set1 $a_{i i}=0$.

Assume we are given a labeled chord diagram. Define the surgery over the set of chords as follows. For every chord, we draw a parallel chord near it and remove the arc of the circle between adjacent ends of the chords as in Fig. 9. By a small perturbation, the picture in $\mathbb{R}^{2}$ is transformed into one-manifold in $\mathbb{R}^{3}$. This manifold $m(D)$ is the result of surgery, see Fig. 10.

Surprisingly, the number of the connected components of $m(D)$ can be counted out of the intersection graph.

\footnotetext{
${ }^{1}$ The case $a_{i i}=1$ corresponds to framed chords with framing 1 , which, in turn, correspond to non-orientable atoms, that we shall consider in another paper.
} 


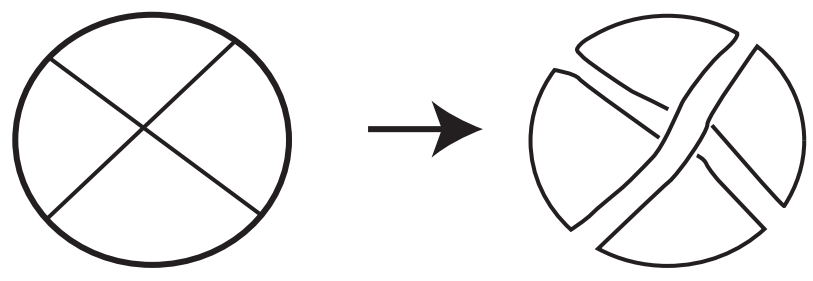

Figure 10: The manifold $m(D)$

Theorem 2.5 ([Sob], $[\mathrm{BNG}]$ ). Let $D$ be a (labeled) chord diagram, and let $G$ be its (labeled) intersection graph. Then the number of connected components of $m(D)$ equals corank $A(G)+1$, where $A(G)$ is the adjacency matrix of $G$.

Remark 2.9. Theorems 2.5 allows us to define 'the number of circles' for a graph even when the given graph is not a intersection graph.

\subsection{Acknowledgments}

The authors are grateful to L. H. Kauffman, V. A. Vassiliev, and A. T. Fomenko for their interest to this work.

\section{The Kauffman bracket polynomial}

The Kauffman bracket polynomial Ka1, Ka2 is a very useful model for understanding the Jones polynomial [Jon]. The Kauffman bracket polynomial associates with a virtual link diagram a Laurent polynomial in one variable $a$. After a small normalisation (multiplication by a power of $(-a)$ ) it gives an invariant for virtual links.

This invariant can be read out of the atom corresponding to a knot diagram. Namely, take an atom $V$ with $n$ vertices corresponding to a virtual diagram $L$ with $n$ classical crossings. By a state we mean a choice of a pair of black or white angles at every vertex of $V$. Every such choice gives rise to a collection of closed curves on $V$ whose boundaries contain all edges of $V$, see Fig. 11, and at each crossing the curves turn locally from one edge to an adjacent edge sharing the same angle of the prefixed colour.

Thus, having $2^{n}$ states of the atom, we define the Kauffman bracket polynomial of it as

$$
\langle V\rangle=\sum_{s} a^{\alpha(s)-\beta(s)}\left(-a^{2}-a^{-2}\right)^{\gamma(s)-1},
$$

where the sum is taken over all states $s$ of the diagram, $\alpha(s)$ and $\beta(s)$ denote the number of white and black angles in the state (thus, $\alpha(s)+\beta(s)=n$ and $\gamma(s)$ denotes the number of curves in the state). 

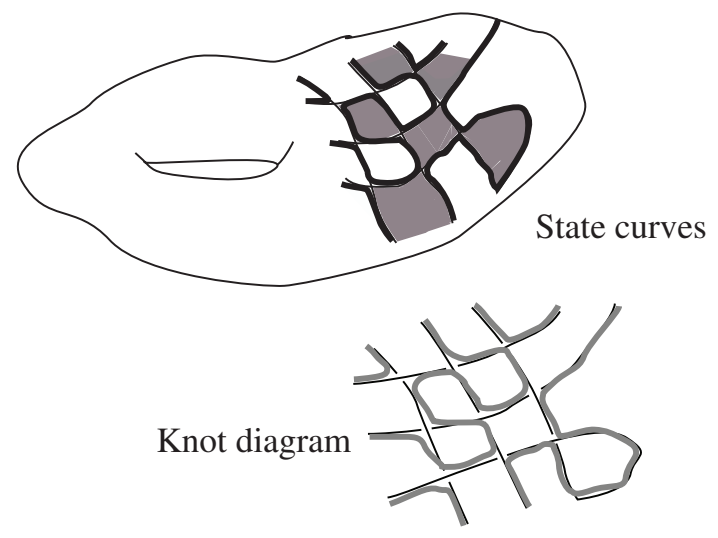

Figure 11: State curves drawn on an atom

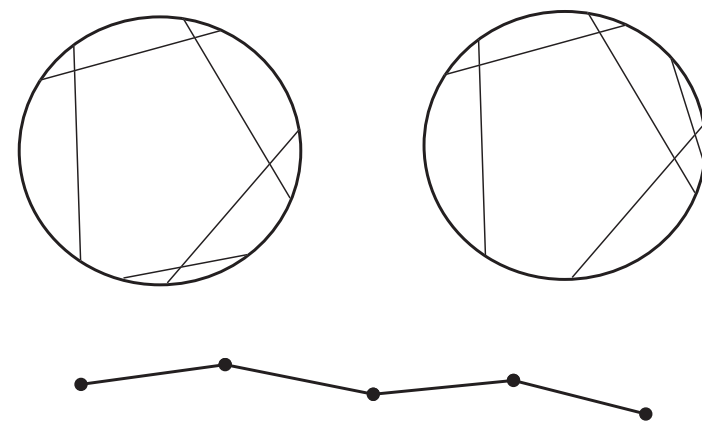

Figure 12: Two chord diagrams with the same intersection graph

As mentioned above, the Kauffman bracket polynomial is invariant under the virtualisation. Thus, it is not surprising that it can be read out of the corresponding atom.

If the atom $A$ is obtained from a (framed) chord diagram $C$, then one can construct the Kauffman bracket polynomial $\langle C(A)\rangle$.

Thus, one obtains a function $f$ on (framed) chord diagrams valued in Laurent polynomials in $a$. We shall return to that function because it is connected to the Vassiliev invariants of knots and $J$-invariants of closed curves (Lando, [Lando]).

Throughout the paper, we consider 'intersection-type' graphs without loops and multiple edges (strict graphs). Assume now we have a labeled graph (a graph with each vertex labeled either positively or negatively).

Then it may or may not be represented as an intersection graph of a chord diagram (see [Bou for the details) for which it is an intersection graph. Moreover, if such a chord diagram exists, it should not be unique, see, e.g., Fig. 12 .

This non-uniqueness usually corresponds to so called mutations of virtual knots. 

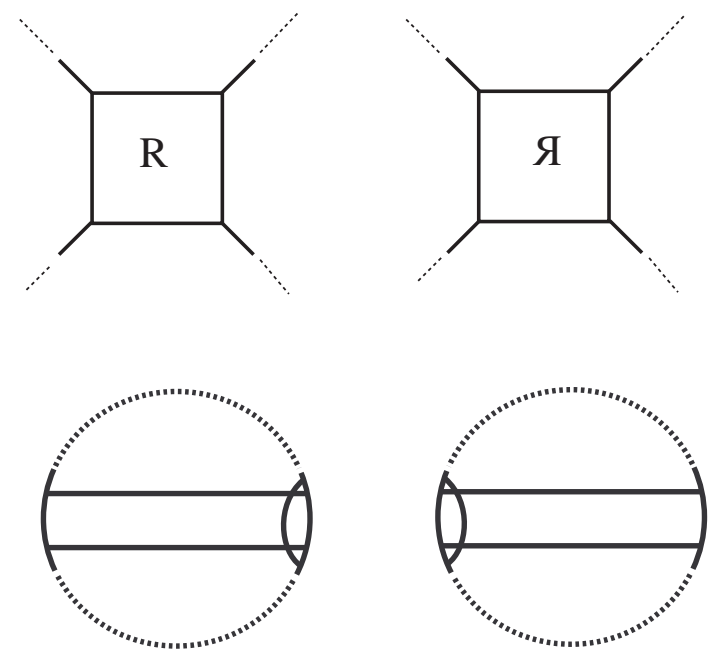

Figure 13: Mutation and its chord diagram presentation

The mutation operation (shown in the top of Fig. 13) cuts a piece of a knot diagram inside a box, turns it by a half-twist and returns to the initial position. Note that the virtualisation is a special case of the mutation.

It turns out that the mutation operation is expressed in terms of chord diagrams in almost the same way: one cuts a piece of diagram with 4 ends and exchanges the top and the bottom parts of it (see bottom picture of Fig. 13). Exactly this operation corresponds to the mutation from both Gauss diagram and rotating circuit points of view.

In the bottom part of Fig. 13 chords whose end points belong to the 'dotted' area remain the same; the other chords (having their end-points in the 'dotted' segments) are reflected as a whole.

It is well-known that the Kauffman bracket polynomial does not detect mutations, see, e.g., Ma4]. Thus, one might guess that the corresponding Kauffman bracket polynomial can be read out of the intersection graph.

Surprisingly, the Kauffman bracket polynomial can be defined (the initial definition was given in [Ma2], but graph-links and Reidemeister moves for graphs were not defined and there was no approach to prove any invariance) in a meaningful way even for those labeled graphs which can not be represented as intersection graphs of chord diagrams.

As we are able to calculate how many circles we have in each state, we can apply (1) to calculate the Kauffman bracket polynomial.

Definition 3.1. Let $G$ be a (strict) labeled finite graph with the set of vertices $V(G)$ and the set of edges $E(G)$. Suppose $s \subset V(G)$. Set $G(s)$ to be the subgraph of the graph $G$ with the set of vertices $V(G(s))=s$ and the set of edges $E(G(s))$ such that $\{u, v\} \in E(G(s))$, where $u, v \in s$, iff $\{u, v\} \in E(G)$. 
Definition 3.2. We call a subset of $V(G)$ a state of the graph $G$. A state is called the $A$-state if it consists of all the vertices of $G$ labeled '-' and no vertex labeled ' + '. Analogously, a state is called the $B$-state if it consists of all vertices of $G$ labeled ' + ' and no vertex labeled '-'. The opposite state for a state $s$ is the set of vertices complement to $s$ (the opposite state to the $A$-state is the $B$-state). Two states are called neighbouring if they differ only in one vertex, which belongs to one state and no to other. The distance between two states is equal to the number of the vertices in which two states differ. A state $s$ is called single-circle state if corank $A(G(s))=0$. We define the number of circles in a state $s$ as corank $A(G(s))+1$.

Remark 3.1. Note that $k+l \leqslant n+2$, here $k$ and $l$ are the numbers of circles in $A$-state and $B$-state, respectively.

Definition 3.3. Let $G$ be a strict finite labeled graph with the set of vertices $V(G)$, $|V(G)|=n$. Define the Kauffman bracket polynomial of $G$ as

$$
\langle G\rangle=\sum_{s} a^{\alpha(s)-\beta(s)}\left(-a^{2}-a^{-2}\right)^{\operatorname{corank} A(G(s))},
$$

where the sum is taken over all states $s$ of the graph $G, \alpha(s)$ is equal to the sum of the vertices labeled '-' from $s$ and of the vertices labeled '+' from $V(G) \backslash s, \beta(s)=n-\alpha(s)$. Recall that all matrices are over $\mathbb{Z}_{2}$ and corank $A_{n \times n}=n-\operatorname{rank} A$.

\section{Graph-links}

One can construct the intersection graph from a chord diagram. Therefore we can define graph-moves corresponding the chord-moves. As a result we have a new object - an equivalence class of labeled graphs over formal moves.

Definition 4.1. Let $G$ be a graph and let $v$ be its vertex. The set of all vertices adjacent to $v$ is called environment of a vertex and denoted by $N(v)$ or $N_{G}(v)$.

Definition 4.2. $\Omega_{g}$. The first Reidemeister graph-move is an addition/removal of an isolated vertex labeled ' + ' or '-'.

$\Omega_{g} 2$. The second Reidemeister graph-move is an addition/removal of two vertices not connected by an edge and having the different signs and the same adjacency with others.

$\Omega_{g} 3$. The third Reidemeister graph-move is defined as follows. Let $u, v, w$ be three vertices of $G$ all having label '-' and $u$ is adjacent in $G$ only to $v$ and $w$. Then we change only the adjacency of $u$ with the vertices $t \in N(v) \backslash N(w) \cup N(w) \backslash N(v)$ (for other pairs of vertices we do not change their adjacency). In addition, we change the labels of $v$ and $w$ to ' + '. The inverse operation is also called the third Reidemeister graph-move. 
$\Omega_{g}$. The fourth graph-move is defined as follows. We take two adjacent vertices $u$ labeled $a$ and $v$ labeled $b, a, b \in\{ \pm 1\}$. Then we change labels of $u$ and $v$ so that the label of $u$ becomes $-b$ and the label of $v$ becomes $-a$. In addition, we change the adjacency for each pair $(t, w)$ of vertices, where $t \in N(u), w \in N(v) \backslash N(u)$ and $t \in N(v), w \in N(u) \backslash N(v)$.

Remark 4.1. The fifth graph-move analogous to the fifth chord-move is as follows. We take a vertex $u$ labeled '-', change its label and add two vertices labeled ' + ' and joined with $u$. This operation is denoted by $\Omega_{g} 5$.

The definition above together with the arguments of Theorem 2.4 yields

Theorem 4.1. Let $K_{1}$ and $K_{2}$ be two connected virtual diagrams with orientable atoms, and $G_{1}$ and $G_{2}$ be two labeled intersection graphs obtained from $K_{1}$ and $K_{2}$, respectively. If $K_{1}$ and $K_{2}$ are equivalent in the class of connected diagrams with orientable atoms then $G_{1}$ is obtained from $G_{2}$ by $\Omega_{g} 1-\Omega_{g} 4$ graph-moves.

We defined the Kauffman bracket polynomial. Our goal is to show that it is invariant under some graph-moves, and then to normalise it in order to get the Jones polynomial. As a result, we get an invariant of graph-links, see ahead. Now we are going to define this object and to check the invariance of the Kauffman bracket polynomial up to some factor.

Definition 4.3. A graph-link is an equivalence of strict labeled graphs modulo $\Omega_{g} 1-\Omega_{g} 4$ graph-moves.

Theorem 4.2. The Kauffman bracket polynomial of a labeled graph is invariant under $\Omega_{g} 2-\Omega_{g} 4$ graph-moves.

Proof. Let $G$ be a labeled graph with the set of vertices $V(G)$ and let $\widetilde{G}$ be a graph obtained from $G$ by some graph-move of the graph-moves $\Omega_{g} 2-\Omega_{g} 4$. $V(\widetilde{G})$ is the set of all vertices of $\widetilde{G}$.

1. Let us consider the second graph-move $\Omega_{g} 2$. Suppose that vertices $u \in V(\widetilde{G})$ labeled '+' and $v \in V(\widetilde{G})$ labeled '-' are added to $G$ to get $\widetilde{G}$. Enumerate all vertices of $G$ by numbers from 1 to $n$, and enumerate all vertices of $\widetilde{G}$ by numbers from 1 to $n+2$ so that the number of $u$ is 1 , the number of $v$ is 2 , and $w \in V(\widetilde{G}) \backslash\{u, v\}$ has number $i \geqslant 3$ if and only if $w$ in $G$ has number $(i-2)$. The adjacency matrix $A(\widetilde{G})$ is

$$
A(\widetilde{G})=\left(\begin{array}{ccc}
0 & 0 & \mathbf{a}^{\top} \\
0 & 0 & \mathbf{a}^{\top} \\
\mathbf{a} & \mathbf{a} & A(G)
\end{array}\right),
$$

where bold characters indicate row and column vectors. 
Let $s \subseteq V(G)$. Then

$$
\operatorname{corank} A(\widetilde{G}(s))=\operatorname{corank} A(G(s)),
$$

$$
\operatorname{corank} A(\widetilde{G}(s \cup\{u\}))=\operatorname{corank} A(\widetilde{G}(s \cup\{v\}))=\operatorname{corank} A(\widetilde{G}(s \cup\{u, v\}))-1
$$

and

$$
\begin{aligned}
&\langle\widetilde{G}\rangle= \sum_{s \subseteq V(G)}\left(a^{2(\alpha(s)+1)-(n+2)}\left(-a^{2}-a^{-2}\right)^{\operatorname{corank} A(\widetilde{G}(s))}+\right. \\
&+a^{2 \alpha(s)-(n+2)}\left(-a^{2}-a^{-2}\right)^{\operatorname{corank} A(\widetilde{G}(s \cup\{u\}))}+ \\
&+ a^{2(\alpha(s)+2)-(n+2)}\left(-a^{2}-a^{-2}\right)^{\operatorname{corank} A(\widetilde{G}(s \cup\{v\}))}+ \\
&+\left.a^{2(\alpha(s)+1)-(n+2)}\left(-a^{2}-a^{-2}\right)^{\operatorname{corank} A(\widetilde{G}(s \cup\{u, v\}))}\right)= \\
&= \sum_{s \subseteq V(G)}\left(a^{2 \alpha(s)-n}\left(-a^{2}-a^{-2}\right)^{\operatorname{corank} A(G(s))}+\right. \\
&\left.+a^{2 \alpha(s)-n}\left(-a^{2}-a^{-2}\right)^{\operatorname{corank} A(\widetilde{G}(s \cup\{u\}))}\left(a^{-2}+a^{2}-a^{2}-a^{-2}\right)\right)=\langle G\rangle,
\end{aligned}
$$

here $\alpha(s)$ is equal to the sum of the vertices labeled '-' from $s$ and of the vertices labeled '+' from $V(G) \backslash s$.

2. Let us consider the third graph-move $\Omega_{g} 3$. There are two versions of the third graphmove we consider only one. Enumerate all vertices of $G$ by numbers from 1 to $n$ so that the label of $u$ is 1 , the label of $v$ is 2 , and the label of $w$ is 3 . The vertices of $\widetilde{G}$ have the same numbers as the vertices of $G$ do. The adjacency matrices $A(G)$ and $A(\widetilde{G})$ are

$$
A(G)=\left(\begin{array}{cccc}
0 & 1 & 1 & \mathbf{0}^{\top} \\
1 & 0 & 0 & \mathbf{a}^{\top} \\
1 & 0 & 0 & \mathbf{b}^{\top} \\
\mathbf{0} & \mathbf{a} & \mathbf{b} & B
\end{array}\right), \quad A(\widetilde{G})=\left(\begin{array}{cccc}
0 & 0 & 0 & (\mathbf{a}+\mathbf{b})^{\top} \\
0 & 0 & 0 & \mathbf{a}^{\top} \\
0 & 0 & 0 & \mathbf{b}^{\top} \\
\mathbf{a}+\mathbf{b} & \mathbf{a} & \mathbf{b} & B
\end{array}\right) .
$$


Let $s \subseteq V=V(G) \backslash\{u, v, w\}$. Then

$$
\begin{gathered}
\operatorname{corank} A(G(s))=\operatorname{corank} A(G(s \cup\{u\}))-1=\operatorname{corank} A(G(s \cup\{u, v\}))= \\
=\operatorname{corank} A(G(s \cup\{u, w\}))=\operatorname{corank} A(\widetilde{G}(s)), \\
\operatorname{corank} A(G(s \cup\{v, w\}))=\operatorname{corank} A(\widetilde{G}(s \cup\{v, w\}))= \\
=\operatorname{corank} A(\widetilde{G}(s \cup\{u, v\}))=\operatorname{corank} A(\widetilde{G}(s \cup\{u, w\}))= \\
=\operatorname{corank} A(\widetilde{G}(s \cup\{u, v, w\}))-1, \\
\operatorname{corank} A(G(s \cup\{v\}))=\operatorname{corank} A(\widetilde{G}(s \cup\{v\})), \\
\operatorname{corank} A(G(s \cup\{w\}))=\operatorname{corank} A(\widetilde{G}(s \cup\{w\})), \\
\operatorname{corank} A(G(s \cup\{u, v, w\}))=\operatorname{corank} A(\widetilde{G}(s \cup\{u\})),
\end{gathered}
$$

and

$$
\begin{gathered}
\langle G\rangle=\sum_{s \subseteq V}\left(a^{2 \alpha(s)-n}\left(-a^{2}-a^{-2}\right)^{\operatorname{corank} A(G(s))}+\right. \\
+a^{2(\alpha(s)+1)-n}\left(\left(-a^{2}-a^{-2}\right)^{\operatorname{corank} A(G(s \cup\{u\}))}+\right. \\
\left.+\left(-a^{2}-a^{-2}\right)^{\operatorname{corank} A(G(s \cup\{v\}))}+\left(-a^{2}-a^{-2}\right)^{\operatorname{corank} A(G(s \cup\{w\}))}\right)+ \\
+a^{2(\alpha(s)+2)-n}\left(\left(-a^{2}-a^{-2}\right)^{\operatorname{corank} A(G(s \cup\{u, v\}))}+\left(-a^{2}-a^{-2}\right)^{\operatorname{corank} A(G(s \cup\{u, w\}))}+\right. \\
\left.\left.+\left(-a^{2}-a^{-2}\right)^{\operatorname{corank} A(G(s \cup\{v, w\}))}\right)+a^{2(\alpha(s)+3)-n}\left(-a^{2}-a^{-2}\right)^{\operatorname{corank} A(G(s \cup\{u, v, w\}))}\right)= \\
=\sum_{s \subseteq V}\left(a^{2 \alpha(s)-n}\left(-a^{2}-a^{-2}\right)^{\operatorname{corank} A(G(s))}\left(1+a^{2}\left(-a^{2}-a^{-2}\right)+2 a^{4}\right)+\right. \\
+a^{2(\alpha(s)+1)-n}\left(\left(-a^{2}-a^{-2}\right)^{\operatorname{corank} A(G(s \cup\{v\}))}+\left(-a^{2}-a^{-2}\right)^{\operatorname{corank} A(G(s \cup\{w\}))}\right)+ \\
+a^{2(\alpha(s)+2)-n}\left(-a^{2}-a^{-2}\right)^{\operatorname{corank} A(G(s \cup\{v, w\}))}+ \\
\left.+a^{2(\alpha(s)+3)-n}\left(-a^{2}-a^{-2}\right)^{\operatorname{corank} A(G(s \cup\{u, v, w\}))}\right)= \\
=\sum_{s \subseteq V}\left(a^{2(\alpha(s)+2)-n}\left(-a^{2}-a^{-2}\right)^{\operatorname{corank} A(G(s))}+\right. \\
+a^{2(\alpha(s)+1)-n}\left(\left(-a^{2}-a^{-2}\right)^{\operatorname{corank} A(G(s \cup\{v\}))}+\left(-a^{2}-a^{-2}\right)^{\operatorname{corank} A(G(s \cup\{w\}))}\right)+ \\
+a^{2(\alpha(s)+2)-n}\left(-a^{2}-a^{-2}\right)^{\operatorname{corank} A(G(s \cup\{v, w\}))}+ \\
\left.+a^{2(\alpha(s)+3)-n}\left(-a^{2}-a^{-2}\right)^{\operatorname{corank} A(G(s \cup\{u, v, w\}))}\right),
\end{gathered}
$$

here $\alpha(s)$ is equal to the sum of the vertices labeled ' - ' from $s$ and of the vertices labeled '+' from $V \backslash s$. 
Analogously for $\widetilde{G}$, we get

$$
\begin{gathered}
\langle\widetilde{G}\rangle=\sum_{s \subseteq V}\left(a^{2(\alpha(s)+2)-n}\left(-a^{2}-a^{-2}\right)^{\operatorname{corank} A(\widetilde{G}(s))}+\right. \\
+a^{2(\alpha(s)+3)-n}\left(-a^{2}-a^{-2}\right)^{\operatorname{corank} A(\widetilde{G}(s \cup\{u\}))}+ \\
+a^{2(\alpha(s)+1)-n}\left(\left(-a^{2}-a^{-2}\right)^{\operatorname{corank} A(\widetilde{G}(s \cup\{v\}))}+\left(-a^{2}-a^{-2}\right)^{\operatorname{corank} A(\widetilde{G}(s \cup\{w\}))}\right)+ \\
+a^{2(\alpha(s)+2)-n}\left(\left(-a^{2}-a^{-2}\right)^{\operatorname{corank} A(\widetilde{G}(s \cup\{u, v\}))}+\left(-a^{2}-a^{-2}\right)^{\operatorname{corank} A(\widetilde{G}(s \cup\{u, w\}))}\right)+ \\
\left.+a^{2 \alpha(s)-n}\left(-a^{2}-a^{-2}\right)^{\operatorname{corank} A(\widetilde{G}(s \cup\{v, w\}))}\right)+ \\
\left.+a^{2(\alpha(s)+1)-n}\left(-a^{2}-a^{-2}\right)^{\operatorname{corank} A(\widetilde{G}(s \cup\{u, v, w\}))}\right)= \\
=\sum_{s \subseteq V}\left(a^{2(\alpha(s)+2)-n}\left(-a^{2}-a^{-2}\right)^{\operatorname{corank} A(G(s))}+\right. \\
+a^{2(\alpha(s)+3)-n}\left(-a^{2}-a^{-2}\right)^{\operatorname{corank} A(G(s \cup\{u, v, w\}))}+ \\
+a^{2(\alpha(s)+1)-n}\left(\left(-a^{2}-a^{-2}\right)^{\operatorname{corank} A(G(s \cup\{v\}))}+\left(-a^{2}-a^{-2}\right)^{\operatorname{corank} A(G(s \cup\{w\}))}\right)+ \\
\left.+a^{2 \alpha(s)-n}\left(-a^{2}-a^{-2}\right)^{\operatorname{corank} A(\widetilde{G}(s \cup\{v, w\}))}\left(2 a^{4}+1+a^{2}\left(-a^{2}-a^{-2}\right)\right)\right)=\langle G\rangle .
\end{gathered}
$$

3. Let us consider the fourth graph-move $\Omega_{g} 4$. Enumerate all vertices of $G$ by numbers from 1 to $n$ as follows. The number of $u$ is 1 , the number of $v$ is 2 , then we enumerate the vertices disjoint with $u$ and $v$, the vertices from $N(u) \backslash N(v)$, the vertices from $N(v) \backslash N(u)$ and the vertices from $N(u) \bigcap N(v)$. We define the numbers of the vertices of $\widetilde{G}$ to be the same as the numbers of the corresponding vertices in $G$. The adjacency matrices $A(G)$ and $A(\widetilde{G})$ are

$$
\begin{gathered}
A(G)=\left(\begin{array}{cccccc}
0 & 1 & \mathbf{0}^{\top} & \mathbf{1}^{\top} & \mathbf{0}^{\top} & \mathbf{1}^{\top} \\
1 & 0 & \mathbf{0}^{\top} & \mathbf{0}^{\top} & \mathbf{1}^{\top} & \mathbf{1}^{\top} \\
\mathbf{0} & \mathbf{0} & A_{0} & A_{1} & A_{2} & A_{3} \\
\mathbf{1} & \mathbf{0} & A_{1} & A_{4} & A_{5} & A_{6} \\
\mathbf{0} & \mathbf{1} & A_{2} & A_{5} & A_{7} & A_{8} \\
\mathbf{1} & \mathbf{1} & A_{3} & A_{6} & A_{8} & A_{9}
\end{array}\right), \\
A(\widetilde{G})=\left(\begin{array}{ccccccc}
0 & 1 & \mathbf{0}^{\top} & \mathbf{1}^{\top} & \mathbf{0}^{\top} & \mathbf{1}^{\top} \\
1 & 0 & \mathbf{0}^{\top} & \mathbf{0}^{\top} & \mathbf{1}^{\top} & \mathbf{1}^{\top} \\
\mathbf{0} & \mathbf{0} & A_{0} & A_{1} & A_{2} & A_{3} \\
\mathbf{1} & \mathbf{0} & A_{1} & A_{4} & A_{5}+(1) & A_{6}+(1) \\
\mathbf{0} & \mathbf{1} & A_{2} & A_{5}+(1) & A_{7} & A_{8}+(1) \\
\mathbf{1} & \mathbf{1} & A_{3} & A_{6}+(1) & A_{8}+(1) & A_{9}
\end{array}\right),
\end{gathered}
$$

where bold characters indicate row and column vectors, (1) is the matrix consisting of 1 , and $A_{j}, j=1, \ldots, 9$, are matrices. 
Let $s \subseteq V(G) \backslash\{u, v\}$. It is not difficult to see that

$$
\begin{gathered}
\operatorname{corank} A(G(s))=\operatorname{corank} A(\widetilde{G}(s \cup\{u, v\})), \\
\text { corank } A(G(s \cup\{u, v\}))=\operatorname{corank} A(\widetilde{G}(s)), \\
\text { corank } A(G(s \cup\{u\}))=\operatorname{corank} A(\widetilde{G}(s \cup\{u\})), \\
\operatorname{corank} A(G(s \cup\{v\}))=\operatorname{corank} A(\widetilde{G}(s \cup\{v\})),
\end{gathered}
$$

and $\alpha(s)$ for $G$ equals $\alpha(s \cup\{u, v\})$ for $\widetilde{G}, \alpha(s \cup\{u, v\})$ for $G$ equals $\alpha(s)$ for $\widetilde{G}$, $\alpha(s \cup\{u\})$ for $G$ equals $\alpha(s \cup\{u\})$ for $\widetilde{G}, \alpha(s \cup\{v\})$ for $G$ equals $\alpha(s \cup\{v\})$ for $\widetilde{G}$. Collecting the terms as above in the Kauffman brackets polynomial of $G$ and $\widetilde{G}$ we immediately get $\langle G\rangle=\langle\widetilde{G}\rangle$, which completes the proof.

Theorem 4.2 yields the following lemma as a corollary.

Lemma 4.1. The Kauffman bracket polynomial of a labeled graph is invariant under $\Omega_{g} 5$ graph-move.

The following theorem describes the difference of the Kauffman bracket polynomials under $\Omega_{g} 1$ graph-move.

Theorem 4.3. The Kauffman bracket polynomial of a labeled graph is multiplied by $\left(-a^{ \pm 3}\right)$ under $\Omega_{g} 1$ graph-move. More precisely, it is multiplied by $\left(-a^{-3}\right)$ under an addition of vertex labeled ' + ' and by $\left(-a^{3}\right)$ under an addition of vertex labeled ' - '.

Proof. The graph $\widetilde{G}$ is obtained from $G$ by addition of one isolated vertex $u$. Enumerate all vertices of $G$ by numbers from 1 to $n$, and enumerate all vertices of $\widetilde{G}$ by numbers from 1 to $n+1$ in such a way that the number of $u$ is 1 and the number of $v \in V(\widetilde{G}) \backslash\{u\}$ is $i \geqslant 2$ if and only if the number of $v$ in $G$ is $i-1$. The adjacency matrix $A(\widetilde{G})$ is

$$
A(\widetilde{G})=\left(\begin{array}{cc}
0 & \mathbf{0}^{\top} \\
\mathbf{0} & A(G)
\end{array}\right)
$$

where bold characters indicate row and column vectors.

Let $s \subseteq V(G)$, we have

$$
\operatorname{corank} A(G(s))=\operatorname{corank} A(\widetilde{G}(s))=\operatorname{corank} A(\widetilde{G}(s \cup\{u\}))-1 .
$$


Suppose the label of $u$ is ' + ', we get

$$
\begin{gathered}
\langle\widetilde{G}\rangle=\sum_{s \subseteq V(G)}\left(a^{2(\alpha(s)+1)-(n+1)}\left(-a^{2}-a^{-2}\right)^{\operatorname{corank} A(\widetilde{G}(s))}+\right. \\
\left.+a^{2 \alpha(s)-(n+1)}\left(-a^{2}-a^{-2}\right)^{\operatorname{corank} A(\widetilde{G}(s \cup\{u\}))}\right)= \\
=\sum_{s \subseteq V(G)} a^{2 \alpha(s)-n}\left(-a^{2}-a^{-2}\right)^{\operatorname{corank} A(G(s))}\left(a+a^{-1}\left(-a^{2}-a^{-2}\right)\right)=-a^{-3}\langle G\rangle,
\end{gathered}
$$

here $\alpha(s)$ is equal to the sum of the vertices labeled '-' from $s$ and of the vertices labeled '+' from $V(G) \backslash s$.

Suppose the label of $u$ is ' - ', we get

$$
\begin{gathered}
\langle\widetilde{G}\rangle=\sum_{s \subseteq V(G)}\left(a^{2 \alpha(s)-(n+1)}\left(-a^{2}-a^{-2}\right)^{\operatorname{corank} A(\widetilde{G}(s))}+\right. \\
\left.+a^{2(\alpha(s)+1)-(n+1)}\left(-a^{2}-a^{-2}\right)^{\operatorname{corank} A(\widetilde{G}(s \cup\{u\}))}\right)= \\
=\sum_{s \subseteq V(G)} a^{2 \alpha(s)-n}\left(-a^{2}-a^{-2}\right)^{\operatorname{corank} A(G(s))}\left(a^{-1}+a\left(-a^{2}-a^{-2}\right)\right)=-a^{3}\langle G\rangle,
\end{gathered}
$$

here $\alpha(s)$ is equal to the sum of the vertices labeled ' - ' from $s$ and of the vertices labeled '+' from $V(G) \backslash s$. This completes the proof.

\section{Graph-knots and the Jones polynomial}

In this section we define graph-knots which are analogous to virtual knots in virtual links, and normalise the Kauffman bracket polynomial, in order to get the Jones polynomial.

To normalise the Kauffman bracket polynomial to an invariant (the Jones polynomial) for the case of links (virtual links), we have to introduce the notion of writhe number (the number of crossings of type $>$ minus the number of crossings of type $>$ ). For a nonoriented knot, this number does not depend on the orientation, whence for a link it does depend on relative orientations of the components. Below we define graph-knots (which correspond to usual knots - one-component links) and define the writhe number and hence the invariant Jones polynomial. A knot is a link having one component, i.e. the set of knots is a proper subset of set of links.

Lemma 5.1. Let $G$ be a labeled graph and $\widetilde{G}$ be a graph obtained from $G$ by some graphmove of $\Omega_{g} 1-\Omega_{g} 4$. If $\operatorname{corank}(A(G)+E)=0$ then $\operatorname{corank}(A(\widetilde{G})+E)=0$, here $E$ is the identity matrix.

Proof. We will use the notations as the same in Theorems 4.2, 4.3, 
1. For the first graph-move $\Omega_{g} 1$ the assertion of the lemma immediately follows from the view of

$$
A(\widetilde{G})=\left(\begin{array}{cc}
0 & \mathbf{0}^{\top} \\
\mathbf{0} & A(G)
\end{array}\right)
$$

2. Consider the second graph-move $\Omega_{g} 2$. Using elementary manipulations with matrices (over $\mathbb{Z}_{2}$ ), we get

$$
\begin{aligned}
A(\widetilde{G})+E & =\left(\begin{array}{ccc}
1 & 0 & \mathbf{a}^{\top} \\
0 & 1 & \mathbf{a}^{\top} \\
\mathbf{a} & \mathbf{a} & A(G)+E
\end{array}\right) \rightsquigarrow\left(\begin{array}{ccc}
1 & 1 & \mathbf{0}^{\top} \\
0 & 1 & \mathbf{a}^{\top} \\
\mathbf{a} & \mathbf{a} & A(G)+E
\end{array}\right) \rightsquigarrow \\
& \rightsquigarrow\left(\begin{array}{ccc}
0 & 1 & \mathbf{0}^{\top} \\
1 & 1 & \mathbf{a}^{\top} \\
\mathbf{a} & \mathbf{a} & A(G)+E
\end{array}\right) \rightsquigarrow\left(\begin{array}{ccc}
0 & 1 & \mathbf{0}^{\top} \\
1 & 0 & \mathbf{0}^{\top} \\
\mathbf{0} & \mathbf{0} & A(G)+E
\end{array}\right)
\end{aligned}
$$

i.e. $\operatorname{corank}(A(\widetilde{G})+E)=\operatorname{corank}(A(G)+E)=0$.

3. Consider the third graph-move $\Omega_{g} 3$. Using elementary manipulations with matrices, we get

$$
\begin{aligned}
& A(\widetilde{G})+E=\left(\begin{array}{cccc}
1 & 0 & 0 & (\mathbf{a}+\mathbf{b})^{\top} \\
0 & 1 & 0 & \mathbf{a}^{\top} \\
0 & 0 & 1 & \mathbf{b}^{\top} \\
\mathbf{a}+\mathbf{b} & \mathbf{a} & \mathbf{b} & B+E
\end{array}\right) \rightsquigarrow\left(\begin{array}{cccc}
1 & 1 & 1 & \mathbf{0}^{\top} \\
0 & 1 & 0 & \mathbf{a}^{\top} \\
0 & 0 & 1 & \mathbf{b}^{\top} \\
\mathbf{a}+\mathbf{b} & \mathbf{a} & \mathbf{b} & B+E
\end{array}\right) \rightsquigarrow \\
& \rightsquigarrow\left(\begin{array}{cccc}
1 & 1 & 1 & \mathbf{0}^{\top} \\
1 & 1 & 0 & \mathbf{a}^{\top} \\
1 & 0 & 1 & \mathbf{b}^{\top} \\
\mathbf{0} & \mathbf{a} & \mathbf{b} & B+E
\end{array}\right)=A(G)+E
\end{aligned}
$$

i.e. $\operatorname{corank}(A(\widetilde{G})+E)=\operatorname{corank}(A(G)+E)=0$.

4. For the fourth graph-move $\Omega_{g} 4$ we have, by using the elementary manipulations with 
matrices,

$$
\begin{aligned}
A(\widetilde{G})+E & =\left(\begin{array}{cccccc}
1 & 1 & \mathbf{0}^{\top} & \mathbf{1}^{\top} & \mathbf{0}^{\top} & \mathbf{1}^{\top} \\
1 & 1 & \mathbf{0}^{\top} & \mathbf{0}^{\top} & \mathbf{1}^{\top} & \mathbf{1}^{\top} \\
\mathbf{0} & \mathbf{0} & A_{0}+E & A_{1} & A_{2} & A_{3} \\
\mathbf{1} & \mathbf{0} & A_{1} & A_{4}+E & A_{5}+(1) & A_{6}+(1) \\
\mathbf{0} & \mathbf{1} & A_{2} & A_{5}+(1) & A_{7}+E & A_{8}+(1) \\
\mathbf{1} & \mathbf{1} & A_{3} & A_{6}+(1) & A_{8}+(1) & A_{9}+E
\end{array}\right) \rightsquigarrow \\
& \rightsquigarrow\left(\begin{array}{cccccc}
1 & 1 & \mathbf{0}^{\top} & \mathbf{1}^{\top} & \mathbf{0}^{\top} & \mathbf{1}^{\top} \\
1 & 1 & \mathbf{0}^{\top} & \mathbf{0}^{\top} & \mathbf{1}^{\top} & \mathbf{1}^{\top} \\
\mathbf{0} & \mathbf{0} & A_{0}+E & A_{1} & A_{2} & A_{3} \\
\mathbf{1} & \mathbf{0} & A_{1} & A_{4}+E & A_{5} & A_{6} \\
\mathbf{0} & \mathbf{1} & A_{2} & A_{5} & A_{7}+E & A_{8} \\
\mathbf{1} & \mathbf{1} & A_{3} & A_{6} & A_{8} & A_{9}+E
\end{array}\right)
\end{aligned}
$$

i.e. $\operatorname{corank}(A(\widetilde{G})+E)=\operatorname{corank}(A(G)+E)=0$, which completes the proof.

Definition 5.1. A graph-link $\{G\}$ is called graph-knot if $\operatorname{corank}\left(A\left(G^{\prime}\right)+E\right)=0$ for any representative $G^{\prime}$ of the graph-link.

Lemma 5.1 guarantees that the notion of graph-knot is well-defined.

The following definition introduces the writhe number for a graph-knot. That number allows us to normalise the Kauffman bracket polynomial.

Definition 5.2. Set the writhe number for a labeled graph $G$ with corank $(A(G)+E)=0$ as follows. Enumerate all vertices of $G$ and $B_{i}(G)=A(G)+E+E_{i i}$ (all elements of $E_{i i}$ except one in the $\mathrm{i}$-th column and $\mathrm{i}$-th row which is equal to 1 are 0 ) for each vertex $v_{i} \in V(G)$. The writhe number $w(G)$ of $G$ is

$$
w(G)=\sum_{i=1}^{n}(-1)^{\operatorname{corank} B_{i}(G)} \operatorname{sign} v_{i} .
$$

Lemma 5.2. The writhe number is an invariant under $\Omega_{g} 2-\Omega_{g} 4$ graph-moves.

Proof. Let $G$ be a graph and $w(G)$ be its writhe number. As above $\widetilde{G}$ is the graph obtained from $G$ by some graph-move. We have $\operatorname{corank}(A(\widetilde{G})+E)=0$ (Lemma 5.1).

We will use the notation as in Theorem 4.2. 
1. Consider the second graph-move $\Omega_{g} 2$. Performing the same elementary manipulation as in Lemma 5.1 we immediately have the equality of coranks of the vertices different from $u$ and $v$.

As

$$
\begin{gathered}
w(\widetilde{G})=\sum_{i=1}^{n+2}(-1)^{\operatorname{corank} B_{i}(\widetilde{G})} \operatorname{sign} w_{i}= \\
=(-1)^{\operatorname{corank} B_{1}(\widetilde{G})} \operatorname{sign} u+(-1)^{\operatorname{corank} B_{2}(\widetilde{G})} \operatorname{sign} v+w(G),
\end{gathered}
$$

then to complete the proof in this case it is sufficient to show that corank $B_{1}(\widetilde{G})=$ corank $B_{2}(\widetilde{G})$. The last fact is obvious.

2. Consider the third graph-move $\Omega_{g} 3$. Performing the same elementary manipulation as in Lemma 5.1 we immediately have the equality of coranks of the vertices different from $u, v$ and $w$.

As

$$
\begin{aligned}
& w(\widetilde{G})=\sum_{i=1}^{n}(-1)^{\operatorname{corank} B_{i}(\widetilde{G})} \operatorname{sign} t_{i}=(-1)^{\operatorname{corank} B_{1}(\widetilde{G})} \operatorname{sign} u+ \\
&+(-1)^{\operatorname{corank} B_{2}(\widetilde{G})} \operatorname{sign} v+(-1)^{\operatorname{corank} B_{3}(\widetilde{G})} \operatorname{sign} v+ \\
&+\sum_{i=4}^{n}(-1)^{\operatorname{corank} B_{i}(\widetilde{G})} \operatorname{sign} t_{i}=(-1)^{\operatorname{corank} B_{1}(\widetilde{G})+1}+ \\
&+(-1)^{\operatorname{corank} B_{2}(\widetilde{G})}+(-1)^{\operatorname{corank} B_{3}(\widetilde{G})}+\sum_{i=4}^{n}(-1)^{\operatorname{corank} B_{i}(G)} \operatorname{sign} t_{i}
\end{aligned}
$$

and

$$
\begin{gathered}
w(G)=\sum_{i=1}^{n}(-1)^{\operatorname{corank} B_{i}(G)} \operatorname{sign} t_{i}=(-1)^{\operatorname{corank} B_{1}(G)} \operatorname{sign} u+ \\
+(-1)^{\operatorname{corank} B_{2}(G)} \operatorname{sign} v+(-1)^{\operatorname{corank} B_{3}(G)} \operatorname{sign} v+ \\
+\sum_{i=4}^{n}(-1)^{\operatorname{corank} B_{i}(G)} \operatorname{sign} t_{i}=(-1)^{\operatorname{corank} B_{1}(G)+1}+(-1)^{\operatorname{corank} B_{2}(G)+1}+ \\
+(-1)^{\operatorname{corank} B_{3}(G)+1}+\sum_{i=4}^{n}(-1)^{\operatorname{corank} B_{i}(G)} \operatorname{sign} t_{i},
\end{gathered}
$$

then to complete the proof in this case it is sufficient to show that corank $B_{1}(\widetilde{G})=$ corank $B_{1}(G)$, corank $B_{2}(\widetilde{G})+\operatorname{corank} B_{3}(G)=1$ and corank $B_{3}(\widetilde{G})+\operatorname{corank} B_{2}(G)=$ 1. 
Let us prove the first and second equalities. We have:

(a)

$$
\begin{aligned}
& B_{1}(\widetilde{G})=\left(\begin{array}{cccc}
0 & 0 & 0 & (\mathbf{a}+\mathbf{b})^{\top} \\
0 & 1 & 0 & \mathbf{a}^{\top} \\
0 & 0 & 1 & \mathbf{b}^{\top} \\
\mathbf{a}+\mathbf{b} & \mathbf{a} & \mathbf{b} & B+E
\end{array}\right) \rightsquigarrow\left(\begin{array}{cccc}
0 & 1 & 1 & \mathbf{0}^{\top} \\
0 & 1 & 0 & \mathbf{a}^{\top} \\
0 & 0 & 1 & \mathbf{b}^{\top} \\
\mathbf{a}+\mathbf{b} & \mathbf{a} & \mathbf{b} & B+E
\end{array}\right) \rightsquigarrow \\
& \rightsquigarrow\left(\begin{array}{cccc}
0 & 1 & 1 & \mathbf{0}^{\top} \\
1 & 1 & 0 & \mathbf{a}^{\top} \\
1 & 0 & 1 & \mathbf{b}^{\top} \\
\mathbf{0} & \mathbf{a} & \mathbf{b} & B+E
\end{array}\right)=B_{1}(G)
\end{aligned}
$$

i.e. $\operatorname{corank} B_{1}(\widetilde{G})=\operatorname{corank} B_{1}(G)$;

(b)

$$
1=\operatorname{det}(A(G)+E)=\operatorname{det} B_{2}(G)+\operatorname{det}\left(\begin{array}{cccc}
1 & 1 & 1 & \mathbf{0}^{\top} \\
0 & 1 & 0 & \mathbf{0}^{\top} \\
1 & 0 & 1 & \mathbf{b}^{\top} \\
\mathbf{0} & \mathbf{a} & \mathbf{b} & B+E
\end{array}\right)
$$

and

$$
\begin{aligned}
& \left(\begin{array}{cccc}
1 & 1 & 1 & \mathbf{0}^{\top} \\
0 & 1 & 0 & \mathbf{0}^{\top} \\
1 & 0 & 1 & \mathbf{b}^{\top} \\
\mathbf{0} & \mathbf{a} & \mathbf{b} & B+E
\end{array}\right) \rightsquigarrow\left(\begin{array}{cccc}
1 & 0 & 0 & \mathbf{0}^{\top} \\
0 & 1 & 0 & \mathbf{0}^{\top} \\
0 & 0 & 0 & \mathbf{b}^{\top} \\
\mathbf{0} & \mathbf{0} & \mathbf{b} & B+E
\end{array}\right) \\
B_{3}(\widetilde{G})= & \left(\begin{array}{cccc}
1 & 0 & 0 & (\mathbf{a}+\mathbf{b})^{\top} \\
0 & 1 & 0 & \mathbf{a}^{\top} \\
0 & 0 & 0 & \mathbf{b}^{\top} \\
\mathbf{a}+\mathbf{b} & \mathbf{a} & \mathbf{b} & B+E
\end{array}\right) \rightsquigarrow\left(\begin{array}{cccc}
0 & 1 & 0 & \mathbf{0}^{\top} \\
1 & 0 & 0 & \mathbf{a}^{\top} \\
0 & 0 & 0 & \mathbf{b}^{\top} \\
\mathbf{0} & \mathbf{a} & \mathbf{b} & B+E
\end{array}\right) \rightsquigarrow \\
& \rightsquigarrow\left(\begin{array}{cccc}
0 & 1 & 0 & \mathbf{0}^{\top} \\
1 & 0 & 0 & \mathbf{0}^{\top} \\
0 & 0 & 0 & \mathbf{b}^{\top} \\
\mathbf{0} & \mathbf{0} & \mathbf{b} & B+E
\end{array}\right)
\end{aligned}
$$

i.e. $1=\operatorname{corank} B_{3}(\widetilde{G})+\operatorname{corank} B_{2}(G)$.

3. Consider the fourth graph-move $\Omega_{g} 4$. Performing the same elementary manipulation as in Lemma 5.1 we immediately have the equality of coranks of the vertices different from $u, v$. 
As

$$
\begin{gathered}
w(\widetilde{G})=\sum_{i=1}^{n}(-1)^{\operatorname{corank} B_{i}(\widetilde{G})} \operatorname{sign} w_{i}=(-1)^{\operatorname{corank} B_{1}(\widetilde{G})} \operatorname{sign} u+ \\
+(-1)^{\operatorname{corank} B_{2}(\widetilde{G})} \operatorname{sign} v+\sum_{i=3}^{n}(-1)^{\operatorname{corank} B_{i}(\widetilde{G})} \operatorname{sign} w_{i}= \\
=(-1)^{\operatorname{corank} B_{1}(\widetilde{G})+1} \operatorname{sign} u+(-1)^{\operatorname{corank} B_{2}(\widetilde{G})+1} \operatorname{sign} v+\sum_{i=3}^{n}(-1)^{\operatorname{corank} B_{i}(G)} \operatorname{sign} w_{i}
\end{gathered}
$$

and

$$
\begin{gathered}
w(G)=\sum_{i=1}^{n}(-1)^{\operatorname{corank} B_{i}(G)+1} \operatorname{sign} w_{i}=(-1)^{\operatorname{corank} B_{1}(G)+1} \operatorname{sign} u+ \\
+(-1)^{\operatorname{corank} B_{2}(G)+1} \operatorname{sign} v+\sum_{i=3}^{n}(-1)^{\operatorname{corank} B_{i}(G)+1} \operatorname{sign} w_{i},
\end{gathered}
$$

then to complete the proof in this case it is sufficient to show that corank $B_{1}(\widetilde{G})+$ corank $B_{1}(G)=1$ and corank $B_{2}(\widetilde{G})+$ corank $B_{2}(G)=1$.

Let us prove only the first equality. We have:

$$
=\operatorname{det} B_{1}(G)+\operatorname{det}\left(\begin{array}{cccccc}
1 & 0 & \mathbf{0}^{\top} & \mathbf{0}^{\top} & \mathbf{0}^{\top} & \mathbf{0}^{\top} \\
1 & 1 & \mathbf{0}^{\top} & \mathbf{0}^{\top} & \mathbf{1}^{\top} & \mathbf{1}^{\top} \\
\mathbf{0} & \mathbf{0} & A_{0}+E & A_{1} & A_{2} & A_{3} \\
\mathbf{1} & \mathbf{0} & A_{1} & A_{4}+E & A_{5} & A_{6} \\
\mathbf{0} & \mathbf{1} & A_{2} & A_{5} & A_{7}+E & A_{8} \\
\mathbf{1} & \mathbf{1} & A_{3} & A_{6} & A_{8} & A_{9}+E
\end{array}\right),
$$


and

$$
\begin{aligned}
B_{1}(\widetilde{G})= & \left(\begin{array}{cccccc}
0 & 1 & \mathbf{0}^{\top} & \mathbf{0}^{\top} & \mathbf{1}^{\top} & \mathbf{1}^{\top} \\
1 & 1 & \mathbf{0}^{\top} & \mathbf{1}^{\top} & \mathbf{0}^{\top} & \mathbf{1}^{\top} \\
\mathbf{0} & \mathbf{0} & A_{0}+E & A_{1} & A_{2} & A_{3} \\
\mathbf{0} & \mathbf{1} & A_{1} & A_{4}+E & A_{5}+(1) & A_{6}+(1) \\
\mathbf{1} & \mathbf{0} & A_{2} & A_{5}+(1) & A_{7}+E & A_{8}+(1) \\
\mathbf{1} & \mathbf{1} & A_{3} & A_{6}+(1) & A_{8}+(1) & A_{9}+E
\end{array}\right) \rightsquigarrow \\
& \rightsquigarrow\left(\begin{array}{cccccc}
0 & 1 & \mathbf{0}^{\top} & \mathbf{0}^{\top} & \mathbf{0}^{\top} & \mathbf{0}^{\top} \\
1 & 1 & \mathbf{0}^{\top} & \mathbf{0}^{\top} & \mathbf{1}^{\top} & \mathbf{1}^{\top} \\
\mathbf{0} & \mathbf{0} & A_{0}+E & A_{1} & A_{2} & A_{3} \\
\mathbf{0} & \mathbf{1} & A_{1} & A_{4}+E & A_{5} & A_{6} \\
\mathbf{1} & \mathbf{0} & A_{2} & A_{5} & A_{7}+E & A_{8} \\
\mathbf{1} & \mathbf{1} & A_{3} & A_{6} & A_{8} & A_{9}+E
\end{array}\right)
\end{aligned}
$$

i.e. $1=\operatorname{corank} B_{1}(\widetilde{G})+\operatorname{corank} B_{1}(G)$, which completes the proof.

The following lemma is evident.

Lemma 5.3. The writhe number is changed by $( \pm 1)$ under $\Omega_{g} 1$ graph-move. More precisely, it is changed by $(-1)$ under an addition of vertex labeled ' + ' and by $(+1)$ under an addition of vertex labeled '-'.

Definition 5.3. Let $G$ be a graph-knot. Define the Jones polynomial as $X(G)=$ $(-a)^{-3 w(G)}\langle G\rangle$.

Theorems 4.2, 4.3 and Lemmas 5.2, 5.3 immediately yield

Theorem 5.1. The Jones polynomial $X(G)$ is an invariant of graph-knots.

\section{Minimal representatives of graph-links}

One of important problems in classical knot theory is to establish minimal crossing number of a certain link. In late 19's century, famous physicist and knot tabulator P. G. Tait [Tait] conjectured that alternating prime diagrams of classical links are minimal with respect to the number of classical crossings. This celebrated conjecture was solved only in 1987, after the notions of the Jones polynomial and the Kauffman bracket polynomial appeared. The first solution was obtained by Murasugi [Mur], then it was reproved by Thistlethwaite [Thi1], Turaev [Tu], and others. Later, Thistlethwaite [Thi2] established the minimality for a larger class of diagrams (so-called adequate diagrams). It turns out that many 
results in these directions generalise for virtual links (establishing the minimal number of classical crossings); these results were obtained by the second named author of the present paper. See [Ma4 for the proofs and some further generalisations and other results concerning virtual knots.

Definition 6.1. The difference between the leading degree and the lowest degree of nonzero terms of the Kauffman bracket polynomial $\langle K\rangle$ is called the span of the Kauffman bracket and is denoted by $\operatorname{span}\langle K\rangle$.

The main reason of these minimality theorems and further crossing estimates come from the well-known Kauffman-Murasugi-Thistlethwaite Theorem:

Theorem 6.1. For a non-split classical link diagram $K$ on $n$ crossings we have $\operatorname{span}\langle K\rangle \leqslant$ $4 n$, whence for alternating non-split diagrams we have $\operatorname{span}\langle K\rangle=4 n$.

Note that the span of the Kauffman bracket is invariant under all Reidemeister moves.

In [Ma4, this theorem is generalised for virtual diagrams. The estimate $\operatorname{span}\langle K\rangle \leqslant 4 n$ can be sharpened to $\operatorname{span}\langle K\rangle \leqslant 4 n-4 g$, where $g$ is the genus of the atom. A nice way to reprove the latter estimate can be also found in [DFKLS], where the authors interpreted atoms as a modification of Grothendieck's dessins d'enfant.

In the present section, we establish minimality of graph-link representatives. We call a labeled graph $G$ minimal if there is no representative of the graph-link corresponding to $G$ having strictly smaller number of vertices than $G$ has.

Definition 6.2. A classical link diagram is called alternating if while passing along every component of it we alternate undercrossings and overcrossings.

From the 'atomic' point of view, alternating link diagrams are those having atom genus (Turaev genus) 0 (more precisely, diagram has genus 0 if it is a connected sum of several alternating diagrams).

For virtual links, we have a notion of quasi-alternating diagram [Ma4]: these are precisely diagrams obtained from classical alternating diagrams by (detours and) virtualisations.

Definition 6.3. A virtual link diagram $D$ is called split if there is a vertex $X$ of the corresponding atom $(M, \Gamma)$ such that $\Gamma \backslash X$ is disconnected.

Now, let us generalise the notions defined above for the case of graph-links.

Definition 6.4. A labeled graph $G$ on $n$ vertices is alternating if $k+l=n+2$, where $k$ is the number of circles in the $A$-state $s_{1}$, i.e. $k=$ corank $A\left(G\left(s_{1}\right)\right)+1$, and $l$ is the number of circles in the $B$-state $s_{2}$ of $G$, i.e. $l=$ corank $A\left(G\left(s_{2}\right)\right)+1$. 
Definition 6.5. A labeled graph $G$ is adequate if the number of circles $k$ in the $A$-state is locally minimal, that is, there is no neighbouring state for the $A$-state with $k+1$ circles, and the same is true for the number of circles $l$ in the $B$-state.

Remark 6.1. This definition of the adequate diagram generalises (see, e.g., [Thi2]) the classical definition of the adequate diagram: no circle of the $A$-state nor $B$-state splits into a pair of circles after one resmoothing.

Definition 6.6. A labeled graph $G$ is non-split if it has no isolated vertices.

Definition 6.7. For a labeled graph $G$ let the atom genus (Turaev genus) be $1-(k+l-$ $n) / 2$, where $k$ and $l$ are the numbers of circles in the $A$-state and $B$-state of $G$, respectively.

Note that this number agrees with the atom genus in the usual case: we just use $\chi=2-2 g$, where $\chi$ is the Euler characteristic, and count $\chi$ by using the number of crossings $n$, number of edges $2 n$ and the number of 2 -cells ( $A$-state circles and $B$-state circles).

Lemma 6.1. For any graph $G$ on $n$ vertices we have $\operatorname{span}\langle G\rangle \leqslant 4 n-4 g(G)$, here $g(G)$ is the genus of the corresponding atom.

Proof. Indeed, the assertion of this lemma comes from the definition of the Kauffman bracket and the atom genus. Denote the number of circles in the $A$-state of $G$ by $k$, and denote the number of circles in the $B$-state of $G$ by $l$. Then the leading term of the Kauffman bracket coming from the $A$-state has degree $n+2(k-1)$, and the lowest term coming from the $B$-state has degree $-n-2(l-1)$. Now, it remains to see that no other state can give a term of degree strictly greater than that of the $A$-state. Similarly, no state contributes a term of degree strictly smaller than that of the $B$-state and the inequality follows.

Lemma 6.2. For an adequate labeled graph $G$ on $n$ vertices we have $\operatorname{span}\langle G\rangle=4 n-$ $4 g(G)$, here $g(G)$ is the genus of the corresponding atom.

Proof. Indeed, it is sufficient to check that the leading term coming from the $A$-state of $G$ is not canceled by any other term coming from another state (the argument for the lowest term coming from the $B$-state is the same).

To do that, let us consider the term $a^{\alpha(s)-\beta(s)}\left(-a^{2}\right)^{\gamma(s)-1}$ for a state $s$. For the $A$ state, we have $\alpha=n, \beta=0, \gamma=k$. If we switch one crossing to the $B$-smoothing, then $\alpha$ is decreased by $1, \beta$ is increased by 1 , which decreases the degree of $a^{\alpha-\beta}$ by two. We may compensate this only if the "number of circles" $\gamma(s)$ (or the corresponding corank $A(G(s))+1$ ) is increased by 1 . This may happen only if there is a state $\widetilde{s}$ adjacent to the $A$-state with corank $A(G(\widetilde{s}))+1=k+1$. Thus, the diagram is inadequate. 
Lemma 6.3. An alternating labeled graph $G$ is adequate if and only if it has no isolated vertices.

Proof. The direction $\Rightarrow$ is obvious. Now, assume that the diagram $G$ is inadequate, alternating and has no isolated vertices. Denote the number of circles of the $A$-state $s_{1}$ by $k$, and that of the $B$-state by $l$. Without loss of generality, assume that there is a state $A^{\prime}$ with $(n-1) A$-smoothings and one $B$-smoothing with number of circles equal to $(k+1)$. Consider the opposite state $B^{\prime}$. Obviously, the number of circles in this state is $l-1$ (the total number can not exceed $k+l$ ). Denote the vertex of $G$ where the $A$-state differs from $A^{\prime}$ by $X$. Thus, the labeled graph $G^{\prime}$ obtained by changing the label of the $X$ has genus 0 , too.

Since $G$ is alternating, all single-circle states are at the same distance from the $A$-state. On the other hand, all single-circle states are at the same distance from $A^{\prime}$. This means that these single-circle states (as subsets of $\{1, \ldots, n\}$ ) either both contain $X$ or both do not contain $X$.

Assume they all contain $X$. We argue that $X$ is an isolated vertex. Indeed, if there were a vertex $Y$ connected to $X$ then, starting from a single-circle state containing $X$ and changing it at $X$ and $Y$, we would get another single-circle state not containing $X$.

This completes the proof.

Lemmas 6.1, 6.2, 6.3 together yield the following

Theorem 6.2. Let $G$ be an alternating labeled graph without isolated vertex. Then it is minimal, that is, there is not graph $G^{\prime}$ with strictly smaller number of vertices representing the same graph-link as $G$.

Proof. Assume the contrary. Then we have $4 n=\operatorname{span}\langle G\rangle=\operatorname{span}\left\langle G^{\prime}\right\rangle \leqslant 4 n^{\prime}-4 g\left(G^{\prime}\right)$, where $n^{\prime}$ is the number crossings of $G^{\prime}$, and $g\left(G^{\prime}\right)$ is the atom genus (Turaev genus) of $G^{\prime}$. The inequality $n^{\prime}<n$ leads to a contradiction, which completes the proof.

Classical links are represented by $d$-diagrams; alternating links are represented by those $d$-diagrams where all chords of one family have sign ' + ' and all chords of the other family have sign '-'. At the level of graphs, $d$-diagrams are bipartite graphs.

Definition 6.8. We call any bipartite graph with arbitrary labeling pseudo-classical.

It is easy to see that a labeled graph is alternating if and only if it is pseudo-classical, and all labels of one subset of disjoint vertices are ' + ' and all labels of the complement subset of vertices (which are pairwise disjoint as well) are '-'.

Example. Consider the graph $G_{7}$ consisting of the 7 vertices with the following incidences. For $i, j=1, \ldots, 6, i$ is connected to $j$ iff $i-j \equiv \pm 1(\bmod 6)$, and 7 is connected 


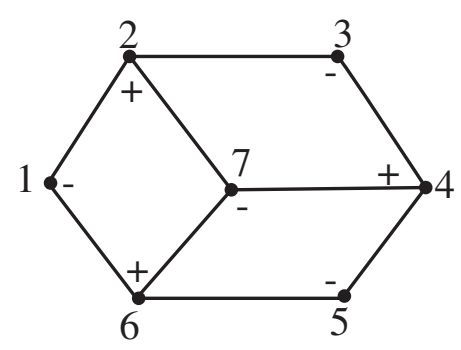

Figure 14: The graph $G_{7}$

to 2, 4,6. Label all even vertices by '+', and label all odd vertices by '-', see Fig. 14. This graph is alternating. By Theorem 6.2, $G_{7}$ is minimal. Note that this graph is not realisable as an intersection graph of a chord diagram, see [Bou. We conjecture that the graph-link represented by $G_{7}$ has no 'realisable' representatives which correspond to classical or virtual diagrams via chord diagrams. At least we know that it has no such representatives with the number of crossings less than or equal to 7 .

\section{References}

[BN] D. Bar-Natan, On the Vassiliev Knot Invariants (1995), Topology, 34, pp. 423-472.

[BNG] D. Bar-Natan and S. Garoufalidis, On the Melvin-Morton-Rozansky conjecture (1996), Inv. Math. 125, pp. 103-133.

[Bou] A. Bouchet, Circle graph obstructions (1994), J. Combinatorial Theory B, 60, pp. 107-144.

[CDL] S. V. Chmutov, S. V. Duzhin, S. K. Lando, Vassiliev Knot Invariants (1994). I,II, III Adv. Sov. Math., 21, pp. 117-147.

[DFKLS] O. Dasbach, D. Futer, E. Kalfagianni, X.-S. Lin and N. Stoltzfus, The Jones polynomial and graphs on surfaces (2008), Journal of Combinatorial Theory, B, 98 (2), pp. 384-399.

[FKM] R. A. Fenn, L. H. Kauffman, V. O. Manturov, Virtual knots - unsolved problems (2006), Fundamenta Mathematicae, N. 188, pp. 293-323.

[Fom] A. T. Fomenko, The theory of multidimensional integrable hamiltonian systems (with arbitrary many degrees of freedom). Molecular table of all integrable systems with two degrees of freedom (1991), Adv. Sov. Math., 6, pp. 1-35.

[GPV] M. Goussarov, M. Polyak, and O. Viro // Topology. 2000. V. 39, pp. 1045-1068. 
[Jon] V.F. R. Jones, A polynomial invariant for links via Neumann algebras (1985), Bull. Amer. Math. Soc., 129, pp. 103-112.

[Ka1] L. H. Kauffman, Virtual knot theory, Eur. J. Combinatorics. 1999. V. 20, N. 7, pp. 662-690.

[Ka2] L. H. Kauffman, State Models and the Jones Polynomial (1987), Topology, 26, pp. 395-407.

[Kh] M. Khovanov, A categorification of the Jones polynomial (1997), Duke Math. J,101 (3), pp. 359-426.

[KhR1] M. Khovanov, L. Rozansky, Matrix Factorizations and Link Homology, arxiv.math: GT/0401268.

[KhR2] M. Khovanov, L. Rozansky, Matrix Factorizations and Link Homology II, arxiv.math: GT/050506.

[Kup] G. Kuperberg, What is a Virtual Link? (2002), www.arxiv.org, math-GT/ 0208039, Algebraic and Geometric Topology, 2003, 3, 5 87-591.

[Lando] S. K. Lando, J-invariants of ornaments and framed chord diagrams (2006), Functional Analysis and Its Applications, 40 (1), pp. 1-13.

[Ma1] V. O. Manturov, Khovanov homology of virtual knots with arbitrary coefficients), Russ. Acad. Sci. Math. Izvestiya, 2007, Vol. 71, N. 5, pp. 967-999.

[Ma2] V.O. Manturov, Embeddings of four-valent framed graphs into 2-surfaces, arxiv.math: GT/0804.4245

[Ma3] V. O. Manturov, Bifurcations, Atoms, and Knots (2000), Moscow Univ. Math. Bull. 1, pp. 3-8.

[Ma4] V. O. Manturov, Teoriya Uzlov (Knot Theory), (Moscow-Izhevsk, RCD), 2005 (512 pp.).

[Mur] K. Murasugi, The Jones polynomial and classical conjectures in knot theory (1987), Topology 26, pp. 187-194.

[Oht] T. Ohtsuki, Quantum Invariants (2002). A Study of Knots, 3-Manifolda, and their Sets, Singapore: World Scientific.

[Sob] E. Soboleva, Vassiliev Knot Invariants Coming from Lie Algebras and 4-Invariants (2001), Journal of Knot Theory and Its Ramifications, 10 (1), pp. 161-169.

[Tait] P. G. Tait, On knots (1898), in Scientific paper I, (London: Campbridge University Press), pp. 273-317.

[Thi1] M. B. Thistlethwaite, Kauffman polynomial and alternating links (1988), Topology, 27, pp. 311--318 
[Thi2] M. B. Thistlethwaite, On the Kauffman polynomial of an adequate link (1988), Invent. Math. 93 (2), pp. 285-296.

[TZ] L. Traldi, L. Zulli, A bracket polynomial for graphs, arxiv.math: GT/0808.3392.

[Tu] V. Turaev, A simple proof of the Murasugi and Kauffman theorems on alternating links (1987), L'Enseignement Mathématique, 33, pp. 203-225.

[Weh] S. Wehrli, Khovanov homology and Conway mutations (2003), arxiv.math: GT/0301312. 